\title{
G

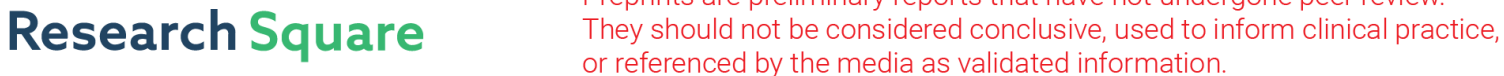

\section{Urinary Metabolomics Revealed the Biological Characteristics Associated with Early Pregnancy in Pigs}

\section{Chen Zhou ( $\nabla$ czhou@stu.scau.edu.cn )}

South China Agricultural University College of Animal Science

\section{Gengyuan Cai}

South China Agricultural University College of Animal Science

\section{Fanming Meng}

Guangdong Academy of Agricultural Sciences

\section{Qun Hu}

South China Agricultural University College of Animal Science

\section{Guohao Liang}

South China Agricultural University College of Animal Science

\section{Ting Gu}

South China Agricultural University College of Animal Science

\section{Enqin Zheng}

South China Agricultural University College of Animal Science

\section{Zicong Li}

South China Agricultural University College of Animal Science

\section{Zhenfang Wu}

South China Agricultural University College of Animal Science

\section{Linjun Hong}

South China Agricultural University College of Animal Science

\section{Research Article}

Keywords: Pig, Early Pregnancy, Urine, Metabolome, Pregnanediol-3-glucuronide

Posted Date: September 17th, 2021

DOI: https://doi.org/10.21203/rs.3.rs-903280/v1

License: (1) (1) This work is licensed under a Creative Commons Attribution 4.0 International License. Read Full License 


\section{Abstract \\ Background}

Accurate early detection of pregnancy status is a prerequisite for effective monitoring of fertility in pig. In the early phase of pregnancy, because the embryo is small and in a free state, it is difficult to determine whether it is pregnant based on B-ultrasound examination. This calls for development of novel tools to accurately diagnose early pregnancy. Metabolomics reveal the metabolic status of cells, tissues and organisms.

\section{Results}

In this study, we investigated urinary metabolites in sows during early pregnancy. A total of 32 samples from 8 sows were collected at estrus and each phase of early pregnancy (days 9, 12, and 15 of gestation). Metabolites in urine samples from different sows obtained from gestation and estrus phases were analyzed via ultra-high performance liquid chromatography/mass spectrometry. A total of 530 metabolites were identified with high confidence in all samples. Compared with samples collected during the estrus phase, 269 differential metabolites were found in samples obtained during early pregnancy.

\section{Conclusions}

These metabolites included lipids and lipid-like molecules, organic acids and their derivatives, organic oxygen compounds, organoheterocyclic compounds, benzenoids, among others. These metabolites, such as choline and pregnanediol-3-glucuronide, play a very important role in pregnancy. They also regulate pregnancy in other animals. Our results provide novel insights into the metabolic changes in the urine of sows in the early pregnancy phase. The level of different metabolites in urine can be used to diagnosis pregnancy in sows. Understanding these metabolic changes is helpful for better management of pregnant sows.

\section{Introduction}

Early pregnancy in pigs is a complex process. Embryo implantation stage (First 15 days of pregnancy), is the phase of early pregnancy in sows. During this phase, the embryo and endometrium undergo strong morphological changes[1, 2]. The embryo moves freely throughout the uterine cavity, and gradually differentiates from a filamentous shape into a fetal shape[3]. During this process, the pig embryo moves between the uterine horns to provide enough space for attachment of the embryo[4]. Through the embryo attachment stage, steroid hormones are secreted which assist in establishing a strong connection between endometrium and the embryo [5]. as well as many different preparations, such as growth factors, prostaglandins, and cytokines[6]. In the free blastocyst phase, embryo-maternal communication is mediated through complex signaling factors. The embryo-maternal, communication is also fed back in 
body fluids including urine as revealed in metabolomic studies[7]. Therefore, it is particularly important to explain the physiological state of pigs in early pregnancy from various aspects.

Metabolomics is the study of metabolites and their chemical processes in biological samples. While a number of metabolites could be use as a source of metabolic information, mammalian urine offers some chief advantages as it contains a large number of metabolites[8] and can be easily collected noninvasively thus minimizing the impact on animals[9]. In addition, as a metabolite of humoral circulation, urine can better reflect all biochemical pathways in the body[10]. In recent years, most clinical assays have applied metabolomics in the study of pregnancy and pregnancy-related diseases like diabetes and ectopic pregnancy[11, 12]. Such studies have been involved use of maternal blood, amniotic fluid, and follicular fluid as metabolites $[13,14]$. Elsewhere, mammalian urine has been used as a biomarker for the study of gestational diabetes mellitus and abortion[12]. Research has hypothesized that activities like pregnancy induce metabolomic signatures that can be observed in mammalian urine[15]. However, there is little knowledge regarding urine metabolomic characteristics associated with early pregnancy phase of mammals especially pigs.

Pig embryos are too small and maternal pregnancy stage is not prominent. It is difficult to diagnose the pregnancy status from the appearance or with the aid of B-ultrasound. Pig embryo implantation in early pregnancy is a multi-complex process which is affected by genetic, nutritional and environmental aspects. The effects arising from these aspects may eventually be reflected in the final metabolites. Therefore, metabolomics analysis of sow urine in early pregnancy is a promising method to identify metabolic biomarkers in early pregnancy. If non-pregnant are detected in early pregnancy phases, then intervention measures can be taken, to better improve reproductive efficiency and economic benefits. The present study used ultra-performance liquid chromatography-mass spectrometry to explore the differential changes in urine metabolites of sows in early pregnancy, and to screen out metabolites that can be potential candidate markers in early pregnancy. Biomarkers revealed in this study can be used to model evidence of maternal-embryo communication as well as regulation and control strategies of maternal nutritional requirements for embryo implantation during early pregnancy in pigs.

\section{Materials And Methods}

\subsection{Study Animals and Sample Collection}

Ten diseases free Large White sows from pig farm were selected for estrus synchronization treatment all together and thereafter artificial insemination was carried out. Urine sample of sows on the 0th (DP0, nonpregnant, calculated on the day before insemination), 9th (DP9), 12th (DP12) and 15th (DP15) day of pregnancy was collected. The collected urine samples were quickly put into liquid nitrogen awaiting metabolite detection[16]. Immediate freezing of samples was necessary to quench any rapid degradation activity such as oxidation of labile metabolites as well as various enzymatic reaction[17, 18]. The pregnancy status of the sows was confirmed with B-ultrasound at the 4th and 6th week. 
Briefly, $200 \mu \mathrm{L}$ urine sample was added into a $1.5 \mathrm{~mL}$ centrifuge tube using a pipette. A $800 \mu \mathrm{L}$ acetonitrile: methanol $(1: 1, \mathrm{v} / \mathrm{v})$ extraction solution was added to the content in the centrifuge tube and then vortex for $30 \mathrm{~s}$ at $5^{\circ} \mathrm{C}$ and $40 \mathrm{kHz}$ Ultrasound for 30 minutes. The samples were placed at $-20^{\circ} \mathrm{C}$ for 30 minutes to precipitate proteins. The supernatant was removed and dried with nitrogen after centrifugation at $13,000 \subseteq \mathrm{g}$ for 15 minutes at $4^{\circ} \mathrm{C}$. Next, reconstitute with $120 \mu \mathrm{L}$ acetonitrile: water $(1: 1$, v/v) reconstituted solution, ultrasonically extract at $5^{\circ} \mathrm{C}$ and $40 \mathrm{kHz}$ for 5 minutes, centrifuge at $13,000 \subseteq \mathrm{g}$ at $4^{\circ} \mathrm{C}$ for 5 minutes, and carefully transfer the supernatant to the sample LC-MS/MS analysis was performed in the bottle[19].

To evaluate stability of analysis system during the on-boarding process, a $20 \mu \mathrm{L}$ supernatant was added to each sample using a pipette and mixed as a quality control sample(Quality Control, QC). During the instrumental analysis, a QC sample was injected for every 8 analytical samples. In data analysis, the repeatability of the QC sample can be used to investigate the stability of the instrument, and find variables with large variations in the analysis system to ensure the reliability of the results[20].

\section{3 (UHPLC-MS/MS) analysis}

The chromatographic separation of metabolites was carried out on a Thermo UHPLC system equipped with an ACQUITY BEH C18 column $(100 \mathrm{~mm} \times 2.1 \mathrm{~mm}$ I.D., Waters, Milford, USA). The mobile phase included $0.1 \%$ formic acid in water (solvent $A$ ) and $0.1 \%$ formic acid in acetonitrile: isopropanol $(1: 1, v / v)$ (solvent $B$ ). To balance the system, the solvent gradient varied according to the following conditions: from 0 to 3 minutes, from 95\% (A): $5 \%$ (B) to $80 \%(A)$ : 20\% (B); from 0 to 3 minutes. 3 to 9 minutes, $80 \%$ (A): $20 \%(B)$ to $5 \%(A): 95 \%(B) ; 9$ to 13 minutes, and 5\% (A): $95 \%(B)$ to $5 \%(A): 95 \%(B)$. From 13 to 13.1 minutes, $5 \%(A)$ : $95 \%(B)$ to $95 \%(A): 5 \%(B)$, from 13.1 to 16 minutes, and $95 \%(A): 5 \%(B)$ to $95 \%(A): 5 \%$ (B). The sample injection volume is $2 \mu \mathrm{L}$, and the flow rate is set to $0.40 \mathrm{~mL} /$ minutes. The column temperature is kept at $40^{\circ} \mathrm{C}$. During the analysis, all these samples were stored at $4{ }^{\circ} \mathrm{C}$.

Mass spectrum data were collected using Thermo UHPLC-Q Exactive Mass Spectrometer equipped with electrospray ionization (ESI) source operating in positive and negative ion mode respectively. The optimum conditions were as follows: Aus gas heater temperature $400^{\circ} \mathrm{C}$, sheath gas flow $40 \mathrm{psi}$; Aus gas flow 30 psi; ion spray voltage floating (ISVF), negative mode-2800V and positive mode 3500V, normalized collision energy, MS / MS rolling 20-40-60V. Data acquisition was performed with the Data Dependent Acquisition (DDA) mode. The detection was carried out in the mass range of 70-1050 m / z.

\subsection{Metabolomics data processing}

After UPLC-TOF/MS analysis, the raw data was imported into Progenesis QI 2.3 (Waters, USA) for peak detection and comparison. The preprocessing results generated a data matrix consisting of retention time (RT), mass-to-charge ratio $(\mathrm{m} / \mathrm{z})$ values and peak intensity. Search was done using biochemical databases (Human Metabolome Database (HMDB) (http://www.hmdb.ca/) and Metlin database (https://metlin.scripps.edu/)), with cumulative mass, MS/MS fragment spectrum and isotope ratio differences Mass spectrometry to identify these metabolic features. For metabolites with MS/MS 
confirmation, only those metabolites with an MS/MS fragment score higher than 30 were considered to be credibly identified. In the Progenesis QI workflow, we considered a confidence score higher than 50 sufficient for assignment of constituents in the iboga extract.

A multivariate statistical analysis was performed using ropls(Version1.6.2) R package from Bioconductor on Majorbio Cloud Platform (https://cloud.majorbio.com). Principle component analysis (PCA) using an unsupervised method was applied to obtain an overview of the metabolic data. Orthogonal partial least squares discriminant analysis (OPLS-DA) was used for statistical analysis to determine the overall metabolic changes between comparable groups. Before OPLS-DA, all metabolite variables were scaled. Effectiveness of the model was evaluated from the model parameters R2 and Q2, which provided information for the interpretability and predictability of the model and avoided risk of over-fitting. Variable Importance in Projection (VIP) was calculated in OPLS-DA model. P-values were estimated with paired Student's t-test on single dimensional statistical analysis. Statistically significant groups with VIP value more than 1 and p-value less than 0.05 were selected. The first 30 different metabolites were selected in the metabolic concentration of each pregnancy period relative to the estrus period. Various metabolites between the two periods were summarized and mapped to their biochemical pathways through metabolic enrichment and pathway analysis based on database search (KEGG, http://www.genomic.jp/kegg/). Scipy.stats(Python package), using Fisher's exact test, was employed to identify statistically significant enrichment pathways.

\section{Results}

\subsection{Untargeted Metabolic Profiling of Urine During Estrus and Early Pregnancy}

To explore the metabolic changes of urine during pregnancy in sows, non-targeted metabolomics analysis was performed. Chromatographic separation spectra exhibited good overlapping when ion chromatograms of each phase samples and quality control samples, in positive ion mode (Fig. 1A) and negative ion mode (Fig. 1B), were analyzed by UPLC/Q-TOF MS. After data pre-processing, 5,327 features were extracted from positive ions. After normalization, 4,660 features with RSD $<30 \%$ accounted for $87.48 \%$ of all QC samples (Table S1); A total of 8,747 features were extracted from negative ions. After normalization, 7,560 features with RSD $<30 \%$ accounted for $86.43 \%$ of all QC samples (Table S2), and the correlation coefficient among the quality control samples was $>0.95$. These results show that there is no significant batch effect on the preprocessed data. Analysis of over 12,220 mass spectra recorded in the total ionization mode revealed 530 compound identifications with high confidence using Progenesis QI (score > 50, Table S3).

The taxonomic information of metabolites obtained from HMDB 4.0 database showed that there are diverse metabolites in urine (Fig. 2). In metabolic concentration (cationic mode and anionic mode), proportions were as follows: Lipids and lipid-like molecules accounted for 28.57\% (132 metabolites), Organic acids and derivatives accounted for $18.83 \%$ ( 87 metabolites), Organic oxygen compounds accounted for $16.45 \%$ (76 metabolites), Organoheterocyclic compounds accounted for $15.37 \%$ (71 
metabolites), Benzenoids accounted for 8.87\% (41 metabolites), Phenylpropanoids and polyketides accounted for $6.49 \%$ (30 metabolites), Nucleosides, nucleotides, and analogues accounted for $1.95 \%$ (9 metabolites ), Organic nitrogen compounds accounted for $1.52 \%$ (7 metabolites), Organooxygen compounds accounted for $1.52 \%$ (7 metabolites), Hydrocarbons accounted for $0.22 \%$ ( 1 metabolites), Lignans, neolignans and related compounds accounted for $0.22 \%$ ( 1 metabolites).

\subsection{Multivariate Statistical Analysis}

We also perform principal component analysis (PCA) on the data to observe the consistency of the experimental samples and quality control samples. The PCA score curve representing QC samples was tightly clustered compared in relation to other samples (Fig. 3 (A, B)). The QC results show that our experiment is stable and the data obtained meets the conditions of subsequent statistical analysis.

Principal component analysis was further used to determine the separation and aggregation of samples between pregnant and non-pregnant sows. The aggregation points indicated high similarity of the observed variables whereas discrete points showed significant difference in the observed variables (VIP $\geq 1$; ratio $\geq 2$ or Ratio $\leq 1 / 2 ; q \leq 0.05$ ). In the positive ion mode, the PCA score showed $54.50 \%$ variation at PC1 and $10.70 \%$ variation at PC2 (Fig. $3 \mathrm{~A}$ ). In the negative ion mode, the PCA score showed $46.80 \%$ variation at PC1 and $14.30 \%$ variation at PC2 (Fig. 3B). The results showed that urine in estrus and early pregnancy had different metabolic characteristics.

The analysis of the samples PCA diagram (Fig. 3) showed that the metabolites of 9 days of pregnancy (DP9), 12 days of pregnancy (DP12) and 15 days of pregnancy (DP15) were clustered together, while the metabolites of estrus (DPO) were clustered together. There was little difference in urine metabolism of sows in early pregnancy, but there was significant difference between pregnancy and estrus. Next, we will focus on changes in metabolites during pregnancy and estrus.

The results of PCA further showed different metabolic characteristics between urine of pregnant sows and that of estrous sows. This indicates that there is a significant separation of urine metabolites in sows during estrus and early pregnancy. PCA only separated between observation groups in the experimental model. Therefore, to identify more specific differences between groups, PLS-DA, a supervised statistical method of discriminant analysis, was employed. The higher the values of PLS-DA model parameters (R2 and Q2), the higher the reliability of PLS-DA model. Comparative analysis of 9 days of pregnancy and estrus: The R2 of PLS-DA model in positive ion mode was 0.819 and Q2 was 0.961 (Figure S1), while in negative ion mode, R2 of PLS-DA model was 0.809 and Q2 was 0.978 (Figure S1). Comparative analysis of 12 days of pregnancy and estrus: The R2 of PLS-DA model in positive ion mode was 0.821 and Q2 was 0.953 (Figure S2), while in negative ion mode, the R2 of PLS-DA model was 0.836 and Q2 was 0.964 (Figure S2). Comparative analysis of 15 days of pregnancy and estrus: The R2 of PLS-DA model in positive ion mode was 0.651 and Q2 was 0.968 (Figure S3), whereas in negative ion mode, the R2 of PLSDA model was 0.765 and Q2 was 0.983 (Figure S3). Generally, results of the PLS-DA model revealed that, both R2 and Q2 were high in early pregnancy. According to the PLS-DA model parameters, the present explanation of the difference in the model is credible. The PLS-DA model was used for follow-up analysis. 
Furthermore, OPLS-DA was used to analyze metabolites in urine during estrus and pregnancy. Comparative analysis of 9 days of pregnancy and estrus, in positive ion mode, showed OPLS-DA parameters as follows: R_2X $=0.667, \mathrm{R} \_2 Y=0.996$ and Q_2 $=0.949$ (Figure S4) whereas the negative ion mode showed OPLS-DA parameters as follows: R_2X $=0.486, R \_2 Y=0.985$ and Q_2 $=0.951$ (Figure S4). Comparative analysis of 12 th days of pregnancy and estrus, in the positive ion mode revealed OPLS-DA parameters as follows: $R \_2 X=0.810, R \_2 Y=0.992, Q \_2=0.946$ (Figure S5), while the negative ion mode showed OPLS-DA parameters as follows: R_2X $=0.765, \mathrm{R} \_2 Y=0.995, \mathrm{Q} \_2=0.961$ (Figure S5).

Comparative analysis of 15 days of pregnancy and the estrus, in the positive ion mode, showed OPLS-DA parameters as follows: R_2X $=0.710, R \_2 Y=0.984, Q \_2=0.948$ (Figure S6). On the other hand, negative ion mode showed OPLS-DA parameters as follows: R_2X $=0.740, R \_2 Y=0.997, Q \_2=0.965$ (Figure S6). In each comparison, 200 permutation tests were used to validate the OPLS-DA model. The intercept of the OPLS-DA model did not reach the over-fitting threshold $(R 2 Y>0.4, Q 2 Y>0.05)$. The OPLS-DA score chart showed a clear separation of urine between estrus and pregnancy samples. All the Q2 values were greater than 0.4 , indicating that the OPLS-DA model used was more reliable and achieved consistent modeling and prediction results.

\subsection{Potential Biomarkers for Differential Metabolites}

Different metabolites were screened by multivariate statistical analysis of the VIP score obtained via OPLS-DA model. Differential metabolites were defined as follow: VIP $\geq 1$; Ratio $\geq 2$ or Ratio $\leq 1 / 2 ; \mathrm{q} \leq$ 0.05. Compared to estrus, there were 186 differential metabolites (Table S4) at 9 days of gestation, 177 differential metabolites (Table S5) at 12 days of gestation and 169 differential metabolites (Table S6) at 15 days of gestation. Significantly different metabolites were used to construct heat maps for unsupervised clustering.. Heat maps were used to define the different levels of metabolites in urine during estrus and pregnancy. Consistent with the OPLS-DA results, significant clustering (Figure S7) was shown in both early pregnancy and during estrus.

The VIP score based on the OPLS-DA model represented the potential of the metabolite as a biomarker and those variables with VIP score greater than 1 were considered important towards the classification model. Top 30 differential metabolites compared to estrus in three phases of early pregnancy were selected for analysis (Fig. 4A-C). Nine metabolites overlapped during early pregnancy and estrus (Fig. 4D). Of the nine, one metabolite, Pregnanediol-3-glucuronide (PdG), was up-regulated in pregnancy compared to estrus. Eight metabolites that included N6-Carbamoyl-L-threonyladenosine, Salicyluric acid, Prostaglandin E3, Marmelolactone A, Caryophyllene epoxide, 3,4,5-trihydroxy-6-[3-(3-

phenylpropanoyl)phenoxy]oxane-2-carboxylic acid, Glucosyl 6-hydroxy-2,6-dimethyl-2E,7-octadienoate, and 6-(2,4-dihydroxybenzoyloxy)-3,4,5-trihydroxyoxane-2-carboxylic acid down-regulated. Significant changes of these metabolites in the early pregnancy can be used to identify the important candidate biomarkers of pregnancy in sows.

3.4 Metabolic Pathways for Metabolite Analysis 
Variables metabolites from DP9 and DP0, DP12 and DP0, DP15 and DP0, were further used for biochemical pathway and enrichment analysis using metabolite set enrichment analysis(MESA). Results of functional enrichment showed a significant shift in expression of molecules associated with amino acid metabolism, lipid metabolism and organic acid production significantly in early pregnancy. At 9 days of pregnancy, major urinary metabolite enrichment pathways of pregnancy compared with estrus were Bile secretion, Phenylalanine metabolism, Drug metabolism - cytochrome P450, Purine metabolism, Choline metabolism in cancer, Cholinergic synapse, Steroid hormone biosynthesis, and Sphingolipid signaling pathway. At 12 days of pregnancy, major urinary metabolite enrichment pathways of pregnancy compared with estrus were Purine metabolism, Alanine, aspartate and glutamate metabolism, Apoptosis, Glycosaminoglycan biosynthesis - chondroitin sulfate / dermatan sulfate, Tyrosine metabolism, Tryptophan metabolism, Bile secretion, Amino sugar and nucleotide sugar metabolism, Sphingolipid signaling pathway. At 15 days of pregnancy, main urinary metabolite enrichment pathways of pregnancy compared with estrus were Phenylalanine, tyrosine and tryptophan biosynthesis, Glycosaminoglycan biosynthesis - chondroitin sulfate / dermatan sulfate, Phenylalanine metabolism, Tyrosine metabolism, Drug metabolism - cytochrome P450, Purine metabolism. In the urinary metabolic pathway, in early pregnancy, shift in amino acid metabolism was mainly observed in biosynthesis and metabolism of phenylalanine, tyrosine and tryptophan. However, the amino acid metabolic pathway also underwent slight changes at different stages, with phenylalanine predominating on day 9 of pregnancy and tyrosine on days 12 and 15 of pregnancy. In terms of lipid metabolism, the main focus in early pregnancy was on 17-beta-Estradiol-3-glucuronide, 2-Methoxy-estradiol-17b 3-glucuronide, 4-oxo-Retinoic acid, 9,10,13TriHOME, Cholestane-3,7,12,25-tetrol-3-glucuronide, Pregnanediol-3-glucuronide, SM(d18:0/16:1(9Z)) and Traumatic Acid. These metabolic changes in early pregnancy may reflect changes in the nutritional needs of the embryo and uterus, as well as the metabolic state of the mother.

\section{Discussion}

The present study employed MS-based approach and biomarker discovery methods to detect urine metabolism through UPLC Q-TOF/MS analysis. Urine from eight sows during estrus and early pregnancy were examined to determine changes in metabolite levels of different pathways associated with early pregnancy. This study identified 269 differential metabolites by UPLC Q-TOF/MS analysis in early pregnancy. A total of 186 different metabolites were found at 9 days of gestation compared to estrus, 177 different metabolites at 12 days of gestation compared to estrus, and 169 different metabolites at 15 days of gestation compared to estrus. These differential metabolites represent changes in various metabolic processes, such as amino acid and lipid metabolism as well as organic acid content. Choline, one of the metabolites is vitamin B. Choline is an essential nutrient for mammals, an important component of phospholipids in cell membranes and one of the raw materials for the body's synthesis of glycosaminoglycans[21]. The metabolite is involved in lipid metabolism, brain development and fetal development[22, 23]. The choline derivative, phosphatidylcholine, is a major constituent of all cell membranes and is required for the biosynthesis of lipoproteins, including very low-density lipoproteins (VLDLs), which facilitate the hepatic export of lipid[24]. 
During pregnancy, choline ingested by the mother is transported through the placenta to the fetus[25]. Studies have confirmed that adequate choline intake during pregnancy is essential for proper fetal development[26]. Supply of maternal choline to the fetus plays an important role in fetal brain development, membrane biosynthesis, and neurotransmission[24]. In a research model involving rats, prenatal choline supplementation preserved sustained memory state of rats and protected offspring from developing memory defects[27]. In pigs, dietary choline deficiency can cause significant changes in plasma choline metabolites at the end of lactation. These changes in the concentration of nutrients such as choline may affect the development of early newborns[28]. Although no examination was performed on natural pregnant sows, we speculate that this is one of the explanations for the increase in urine choline levels in sows during pregnancy. Pregnancy and lactation are periods when maternal reserves of choline are depleted. At the same time, the availability of choline for normal development of the brain is critical because it influences lifelong memory enhancement[29].

Of the various metabolites differentially expressed, Pregnanediol-3-glucuronide (PdG) was highly expressed in all the three phases of early pregnancy. The PdG metabolite has been reported to play an important role in the regulation of mammalian pregnancy. Volkery et al. studied the content of PdG in plasma, saliva, milk and urine of alpaca without pregnancy and throughout pregnancy. It was found that the concentration of PdG in plasma, milk and urine was significantly higher than that in non-pregnant alpaca[30]. It is believed that the ovaries of mammals are luteinized once they are pregnant and the corpus luteum dissolves at the end of pregnancy. Rene et al, characterized the variability of hormonal profiles during the luteal phase in normal cycles. The authors reported that PdG increased with early lutealization, maintained a high level of PdG in the mid-luteal phase, and gradually decreased to a low level in the late luteal phase (luteolysis) in the non-pregnant state[31]. Elsewhere, the occurrence of normal and low luteal PdG level was confirmed as a potential sign of luteal phase abnormality[32]. In a study carried out by Rene Leiva et al. the assessment of urine PdG was also used as an auxiliary means to confirm the effectiveness of ovulation[33]. Muren et al. measured concentrations of PdG in urine and faeces of two pregnant golden monkey females consecutively using an immunoenzymatic method. They documented that its concentration was positively correlated with the number of pregnant embryos[34]. Chandrasekhar et al. used enzyme immunoassay(EIA) against progesterone, PdG, and 5-alpha-pregnane to monitor pregnancy of musk deer. They found that PdG EIA was a highly accurate and sensitive assay compared to other two metabolites assays in detecting pregnancy[35]. In this study, we found that PdG continued to be highly expressed in the urine of sows in the early pregnancy, and these changes showed the same trend as the above-mentioned various body fluid expression trends in the early pregnancy of other mammals. PdG was the main terminal metabolite of progesterone and plays an important role in physiological processes like female menstrual cycle, pregnancy embryogenesis and maternal immune response in some mammals [36]. Therefore, correlated changes in sow urinary PdG levels can be used as a candidate biomarker for early pregnancy.

We analyzed the pathway of total metabolites at each pregnancy phase of early pregnancy. The results showed that tyrosine metabolism, phenylalanine metabolism and tryptophan metabolism play an important role in pregnancy. Liu et al. carried out a longitudinal analysis of urine from 50 healthy 
pregnant women, and their study showed own-regulation of tyrosine metabolism during pregnancy[12]. Tyrosine metabolism can be formed by phenylalanine hydroxylation during pregnancy [37] and these two pathways are also reflected in this study (Fig. 5). It has been reported that maternal tyrosine intake during gestation plays a pre-regulatory role in development of dopaminergic system in offspring after birth[38]. McBride et al. determined the reference ranges for phenylalanine and tyrosine in healthy pregnancy. The authors reported that phenylalanine and tyrosine declined rapidly in early pregnancy and remained relatively stable in mid- and late pregnancy[39]. Tryptophan is an indispensable amino acid in mammalian protein synthesis. Tryptophan metabolism within different tissues is associated with numerous physiological functions. The liver regulates tryptophan homeostasis through degrading tryptophan in excess. Tryptophan degradation into kynurenine by immune cells plays a crucial role in the regulation of immune response during infections, inflammations and pregnancy[40]. Tryptophan increases maternal protein synthesis, fetal growth and development and improves kynurenines inhibition of fetal rejection during gestation in mammals [41]. The mechanism of tryptophan metabolism in mammalian gestation can be achieved through multiple pathways. For instance, kynurenine pathway where tryptophan is converted to kynurenine by the action of indoleamine 2-3 deoxygenase and tryptophan-2,3-double-oxygenase. Kynurenine is then converted to kynurenic acid by the action of kynurenine aminotransferase (KAT). In the case of depression, kynurenic acid may have neuro-protective effects[42]. The tryptophan metabolic pathway also generates 3-hydroxyanthranilic acid (HA), an effective antioxidant, intra-cellularly via kynurenic acid, in the extrahepatic tissue [43]. Further studies have shown that tryptophan and its metabolites are effective in scavenging free radicals (including reactive oxygen and reactive chlorine). Tryptophan and its metabolites can be targeted in the placenta as potent antioxidants[44]. In our study, compared with the estrus period, a variety of differentially expressed metabolites in early pregnancy are enriched in the amino acid metabolism pathway. This change may be required for embryonic development or immune regulation as in other animals. The present study did not exhaust differential metabolites and related pathways thus there is need for more studies to be carried out in this area.

\section{Conclusion}

The urinary metabolomics of sows during estrus and early pregnancy were determined using UHPLC. A total of 269 urinary metabolites that included lipids and organic acids were identified in early pregnancy. In particular, it was found that PdG was consistently highly expressed at each pregnancy phase of early pregnancy and could be regarded as an important candidate biomarker of early pregnancy. To the best of our knowledge, this is the first investigation of urinary metabolic changes in sows during early pregnancy. Our study contributes to the understanding of maternal urinary changes caused by intrauterine embryo implantation during early pregnancy in sows. The study further forms a basis upon which certain molecular mechanisms for maternal-fetal communication of embryo implantation and candidate noninvasive pregnancy diagnostic biomarkers in early pregnancy can be modeled.

\section{Declarations}




\section{Data Availability Statement}

The complete dataset for this study can be accessed here: https://www.ebi.ac.uk/metabolights/MTBLS2135[45]

\section{Ethics Statement}

All procedures involving animals were conducted under a protocol approved by the Ethics Committees of the Laboratory Animal Center of South China Agricultural University (Approval No. SYXK-2014-0136).

\section{Author Contributions}

C.Z., G.C., L.H. and Z.W. designed the study. Q.H., G.L., T.G., and E.Z. collected and treatment samples. F.M., Z.L. and C.Z. conducted the experiments and performed the sequencing analysis. C.Z., G.C., L.H. and Z.W. drafted the manuscript. All authors read and approved the final manuscript.

\section{Funding}

This work was supported by the Guangdong Provincial Promotion Project on Livestock Industry Transformation and Utilization of Livestock Waste (2018XMZC), the National Natural Science Foundation (Grant No. 31802033), the Department of Science and Technology of Guangdong Province (Grant Nos. 2019A1515011835), and the Science \& Technology Planning Project of Guangzhou (Grant No. 201904010434).

\section{Conflict of Interest}

The authors declare that the research was conducted in the absence of any commercial or financial relationships that could be construed as a potential conflict of interest.

\section{Acknowledgments}

We would like to thank Guangdong Wen's pig breeding company for providing help for this research.

\section{References}

1. Keys JL, King GJ: Microscopic examination of porcine conceptus-maternal interface between days 10 and 19 of pregnancy. The American journal of anatomy1990, 188(3):221-238. 
2. Bazer FW, Johnson GA: Pig blastocyst-uterine interactions. Differentiation; research in biological diversity2014, 87(1-2):52-65.

3. Anderson LL: Growth, protein content and distribution of early pig embryos. The Anatomical record1978, 190(1):143-153.

4. Geisert RD, Schmitt RAM: Early embryonic survival in the pig: Can it be improved? Journal of Animal Science2002, 80(E-suppl_1):E54-E65.

5. Spencer TE, Bazer FW: Conceptus signals for establishment and maintenance of pregnancy. Reproductive biology and endocrinology : RB\&E2004, 2:49.

6. Waclawik A, Kaczmarek MM, Blitek A, Kaczynski P, Ziecik AJ: Embryo-maternal dialogue during pregnancy establishment and implantation in the pig. Molecular reproduction and development2017, 84(9):842-855.

7. MANER DF, SAFFAN BD, WIGGINS RA, THOMPSON JD, PREEDY JRK: Interrelationship of Estrogen Concentrations in the Maternal Circulation, Fetal Circulation and Maternal Urine in Late Pregnancy. The Journal of Clinical Endocrinology \& Metabolism1963, 23(5):445-458.

8. Blazenovic I, Kind T, Sa MR, Ji J, Vaniya A, Wancewicz B, Roberts BS, Torbasinovic H, Lee T, Mehta SSet al: Structure Annotation of All Mass Spectra in Untargeted Metabolomics. Anal Chem2019, 91(3):21552162.

9. Wald C: Diagnostics: A flow of information. Nature2017, 551(7679):S48-S50.

10. Khamis MM, Adamko DJ, El-Aneed A: Mass spectrometric based approaches in urine metabolomics and biomarker discovery. Mass spectrometry reviews2017, 36(2):115-134.

11. Bardanzellu F, Fanos V: The choice of amniotic fluid in metabolomics for the monitoring of fetus health - update. Expert review of proteomics2019, 16(6):487-499.

12. Liu X, Wang X, Sun H, Guo Z, Liu X, Yuan T, Fu Y, Tang X, Li J, Sun Wet al: Urinary metabolic variation analysis during pregnancy and application in Gestational Diabetes Mellitus and spontaneous abortion biomarker discovery. Scientific reports2019, 9(1):2605.

13. Orczyk-Pawilowicz M, Jawien E, Deja S, Hirnle L, Zabek A, Mlynarz P: Metabolomics of Human Amniotic Fluid and Maternal Plasma during Normal Pregnancy. PloS one2016, 11(4):e0152740.

14. Karaer A, Tuncay G, Mumcu A, Dogan B: Metabolomics analysis of follicular fluid in women with ovarian endometriosis undergoing in vitro fertilization. Systems biology in reproductive medicine2019, 65(1):39-47. 
15. Wu JQ, Gao YH: Physiological conditions can be reflected in human urine proteome and metabolome. Expert review of proteomics2015, 12(6):623-636.

16. Fernández-Peralbo MA, Luque de Castro MD: Preparation of urine samples prior to targeted or untargeted metabolomics mass-spectrometry analysis. TrAC Trends in Analytical Chemistry2012, 41:7585 .

17. Bernini P, Bertini I, Luchinat C, Nincheri P, Staderini S, Turano P: Standard operating procedures for preanalytical handling of blood and urine for metabolomic studies and biobanks. Journal of biomolecular NMR2011, 49(3-4):231-243.

18. Ryan D, Robards K, Prenzler PD, Kendall M: Recent and potential developments in the analysis of urine: a review. Analytica chimica acta2011, 684(1-2):8-20.

19. Saude EJ, Sykes BD: Urine stability for metabolomic studies: effects of preparation and storage. Metabolomics2007, 3(1):19-27.

20. Want EJ, Wilson ID, Gika H, Theodoridis G, Plumb RS, Shockcor J, Holmes E, Nicholson JK: Global metabolic profiling procedures for urine using UPLC-MS. Nat Protoc2010, 5(6):1005-1018.

21. Blusztajn JK, Slack BE, Mellott TJ: Neuroprotective Actions of Dietary Choline. Nutrients2017, 9(8).

22. Zeisel SH: Choline: critical role during fetal development and dietary requirements in adults. Annual review of nutrition2006, 26:229-250.

23. Niculescu MD, Craciunescu CN, Zeisel SH: Dietary choline deficiency alters global and gene-specific DNA methylation in the developing hippocampus of mouse fetal brains. FASEB journal : official publication of the Federation of American Societies for Experimental Biology2006, 20(1):43-49.

24. Korsmo HW, Jiang X, Caudill MA: Choline: Exploring the Growing Science on Its Benefits for Moms and Babies. Nutrients2019, 11(8).

25. Baumgartner HK, Trinder KM, Galimanis CE, Post A, Phang T, Ross RG, Winn VD: Characterization of choline transporters in the human placenta over gestation. Placenta2015, 36(12):1362-1369.

26. Drews K: [Folate metabolism-epigenetic role of choline and vitamin B12 during pregnancy]. Ginekologia polska2015, 86(12):940-946.

27. Yang Y, Liu Z, Cermak JM, Tandon P, Sarkisian MR, Stafstrom CE, Neill JC, Blusztajn JK, Holmes GL: Protective effects of prenatal choline supplementation on seizure-induced memory impairment. The Journal of neuroscience : the official journal of the Society for Neuroscience2000, 20(22):RC109.

28. Mudd AT, Alexander LS, Johnson SK, Getty CM, Malysheva OV, Caudill MA, Dilger RN: Perinatal Dietary Choline Deficiency in Sows Influences Concentrations of Choline Metabolites, Fatty Acids, and Amino 
Acids in Milk throughout Lactation. The Journal of nutrition2016, 146(11):2216-2223.

29. Zeisel SH: Choline: needed for normal development of memory. Journal of the American College of Nutrition2000, 19(5 Suppl):528S-531S.

30. Volkery J, Gottschalk J, Sobiraj A, Wittek T, Einspanier A: Progesterone, pregnanediol-3-glucuronide, relaxin and oestrone sulphate concentrations in saliva, milk and urine of female alpacas (Vicugna pacos) and their application in pregnancy diagnosis. The Veterinary record2012, 171(8):195.

31. Ecochard R, Bouchard T, Leiva R, Abdulla S, Dupuis O, Duterque O, Garmier Billard M, Boehringer H, Genolini C: Characterization of hormonal profiles during the luteal phase in regularly menstruating women. Fertility and sterility2017, 108(1):175-182 e171.

32. Abdulla SH, Bouchard TP, Leiva RA, Boyle P, Iwaz J, Ecochard R: Hormonal Predictors of Abnormal Luteal Phases in Normally Cycling Women. Frontiers in public health2018, 6:144.

33. Leiva R, McNamara-Kilian M, Niezgoda H, Ecochard R, Bouchard T: Pilot observational prospective cohort study on the use of a novel home-based urinary pregnanediol 3-glucuronide (PDG) test to confirm ovulation when used as adjunct to fertility awareness methods (FAMs) stage 1. BMJ open2019, 9(5):e028496.

34. Muren, Kusuda S, Doi O, Naito H, Hashikawa H: Puberty, ovarian cycle, pregnancy, and postpartum ovulation in captive Sichuan golden monkeys (Rhinopithecus roxellana) based on changes in urinary and fecal gonadal steroid metabolites. Theriogenology2017, 87:179-186.

35. Mithileshwari C, Srivastava T, Kumar V, Kumar A, Umapathy G: Non-invasive assessment of fecal progestagens and pregnancy detection in Himalayan musk deer (Moschus chrysogaster). Theriogenology2016, 85(2):216-223.

36. Chen J, Hou H, Chen H, Luo Y, He Y, Wang H, Deng H, Zhang L, Zhang Y, Liu Het al: Quantitation of serum pregnanediol-3-glucuronide level in different endocrine statuses by LC-MS/MS. Journal of pharmaceutical and biomedical analysis2020, 184:113171.

37. Whittaker PG, Lee $\mathrm{CH}$, Cooper BG, Taylor R: Evaluation of phenylalanine and tyrosine metabolism in late human pregnancy. Metabolism: clinical and experimenta/1999, 48(7):849-852.

38. Santana C, Martin L, Rodriguez Diaz M: Tyrosine ingestion during rat pregnancy alters postnatal development of dopaminergic neurons in the offspring. Brain research1994, 635(1-2):96-102.

39. McBride KL, Pluciniczak J, Rhyand T, Bartholomew D: Phenylalanine and tyrosine measurements across gestation by tandem mass spectrometer on dried blood spot cards from normal pregnant women. Genetics in medicine : official journal of the American College of Medical Genetics2019, 21(8):1821-1826. 
40. Le Floc'h N, Otten W, Merlot E: Tryptophan metabolism, from nutrition to potential therapeutic applications. Amino acids2011, 41(5):1195-1205.

41. Badawy AA: Tryptophan metabolism, disposition and utilization in pregnancy. Bioscience reports2015, 35(5).

42. Savitz J, Drevets WC, Smith CM, Victor TA, Wurfel BE, Bellgowan PS, Bodurka J, Teague TK, Dantzer R: Putative neuroprotective and neurotoxic kynurenine pathway metabolites are associated with hippocampal and amygdalar volumes in subjects with major depressive disorder.

Neuropsychopharmacology : official publication of the American College of Neuropsychopharmacology2015, 40(2):463-471.

43. Pae HO, Oh GS, Lee BS, Rim JS, Kim YM, Chung HT: 3-Hydroxyanthranilic acid, one of L-tryptophan metabolites, inhibits monocyte chemoattractant protein-1 secretion and vascular cell adhesion molecule1 expression via heme oxygenase-1 induction in human umbilical vein endothelial cells.

Atherosclerosis2006, 187(2):274-284.

44. Xu K, Liu G, Fu C: The Tryptophan Pathway Targeting Antioxidant Capacity in the Placenta. Oxidative medicine and cellular longevity2018, 2018:1054797.

45. Haug K, Cochrane K, Nainala VC, Williams M, Chang J, Jayaseelan KV, O'Donovan C: MetaboLights: a resource evolving in response to the needs of its scientific community. Nucleic acids research2020, 48(D1):D440-D444.

\section{Figures}

A

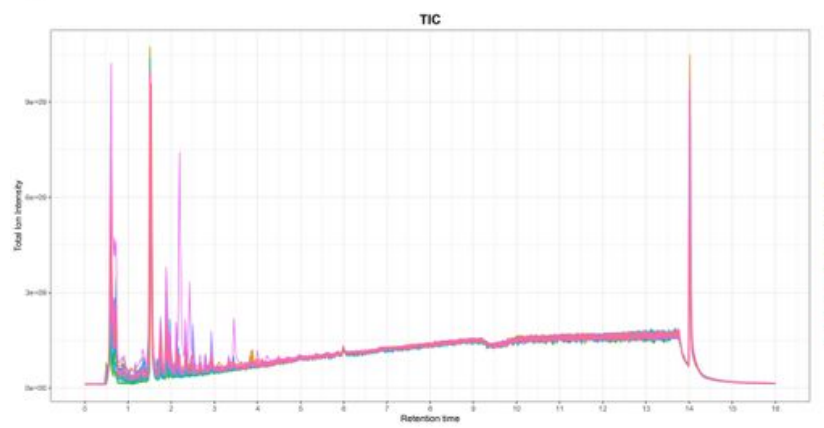

B

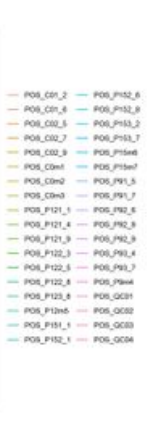

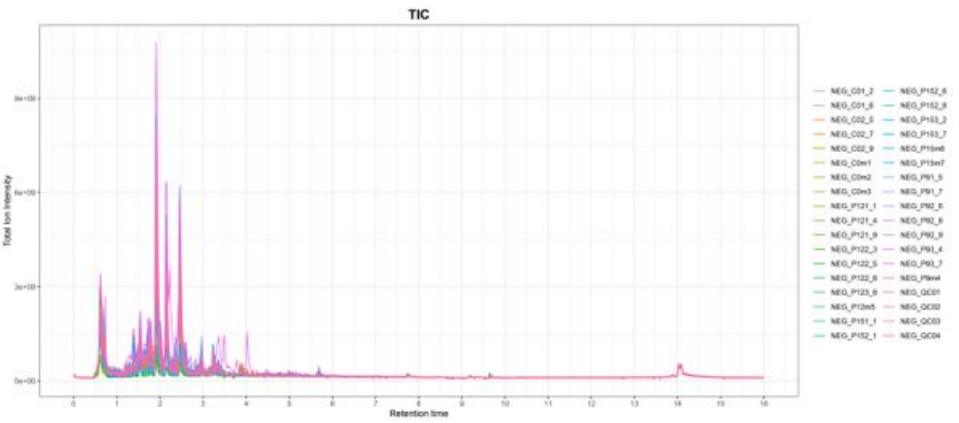

Figure 1

Typical total ion chromatograms (TICs), (A) Positive ions and (B) Negative ions, obtained from the UHPLC-Q Exactive ESI of Quality Control samples. 


\section{Organooxygen com pounds: $1.52 \%$ \\ Organic nitrogen com pounds $: 1.52 \%$}

Lignans, neolignans and related compounds $: 0.22 \%$

Nucleosides, nucleotides, and analogues:1.95\%

Phenylpropanoids and polyketides: $6.49 \%$

Benzenoids : $8.87 \%$

Organoheterocyclic compounds : $15.37 \%$

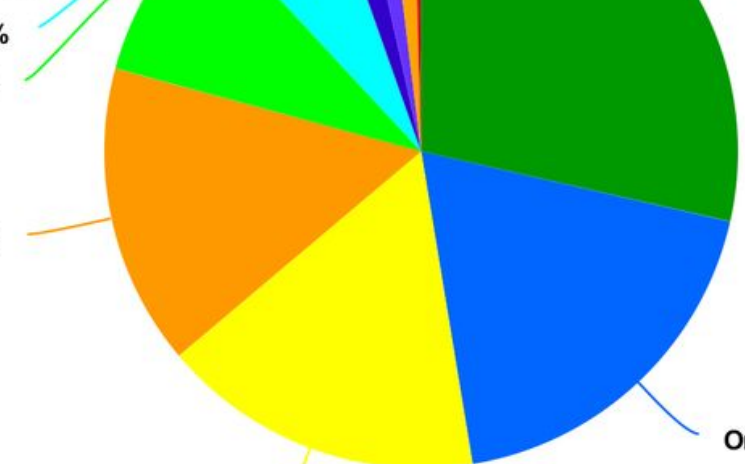

Organic acids and derivatives: $18.83 \%$

Organic oxygen compounds: $16.45 \%$

\section{Figure 2}

Pie chart showing percentage of diverse urine metabolites based on counts of HMDB chemical taxonomy ("Super class"). Name of the selected HMDB level ("Super class") and the percentage of metabolites are displayed in descending order. Different colors on the pie chart represent different HMDB categories and the area represents the relative proportion of metabolites in that category.

A

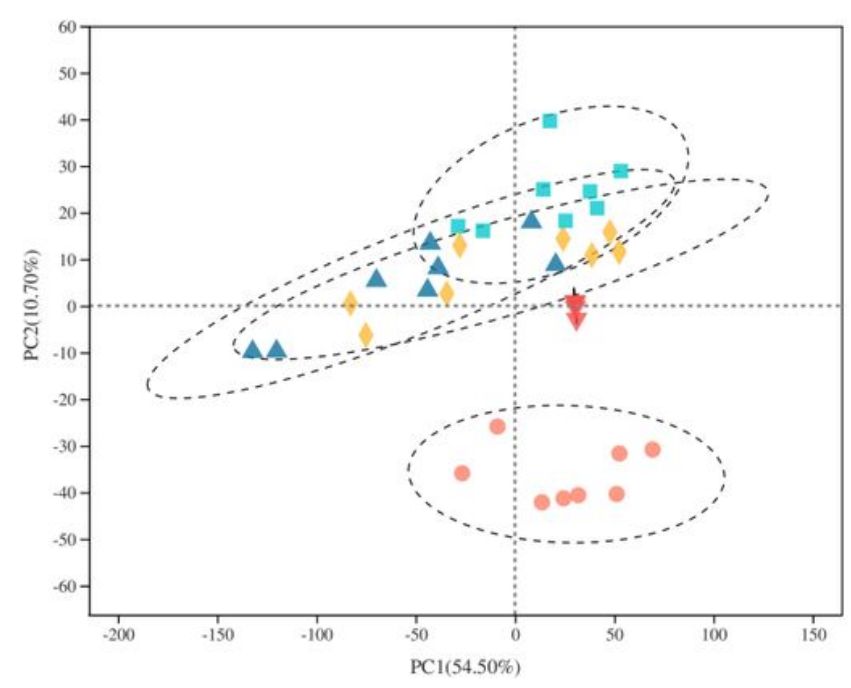

B

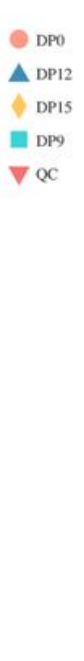

Scores(PCA)

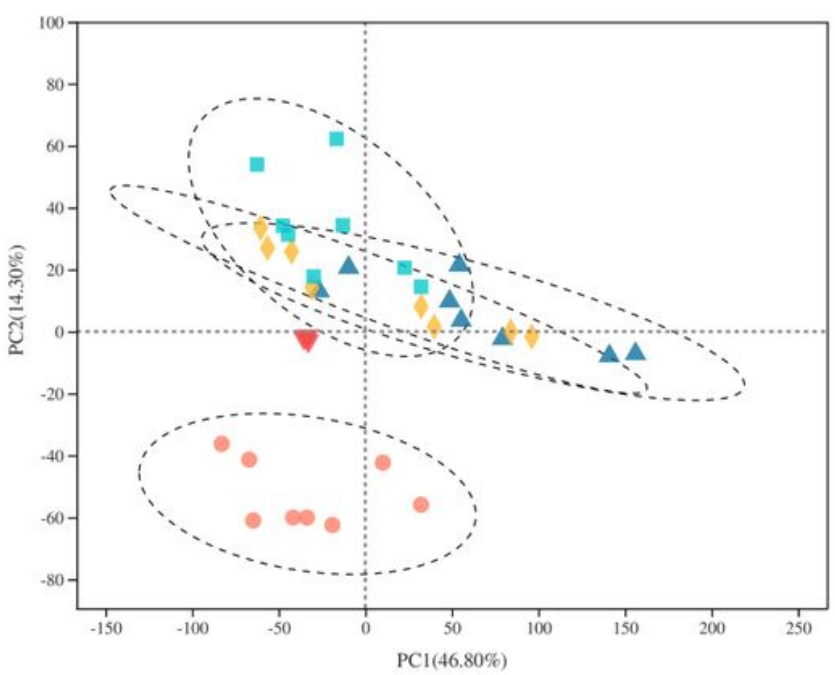

\section{Figure 3}


Principal component analysis score plots of metabolites identified in urine during estrus and pregnancy. Figure (A) represents positive ions, Figure (B) represents negative ions. Each point on the PCA score chart represents a sample.

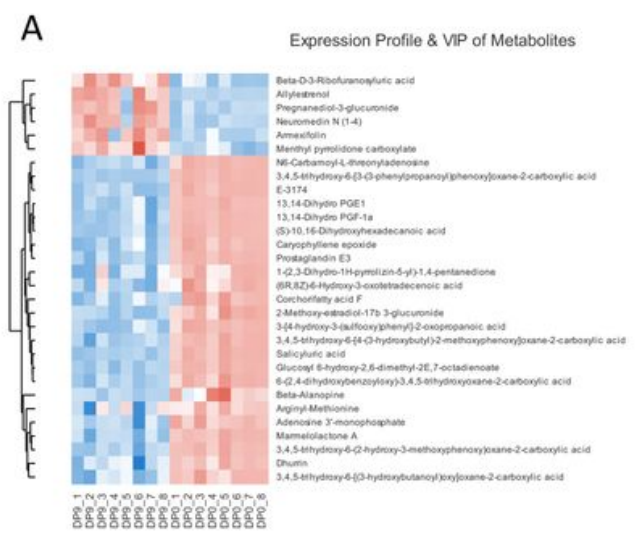

A

C

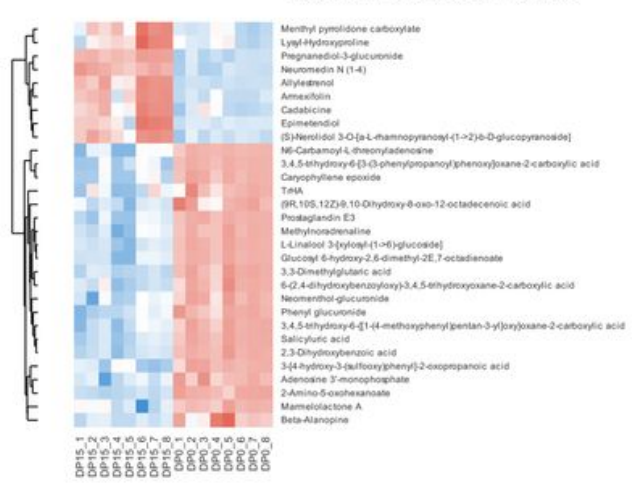

B
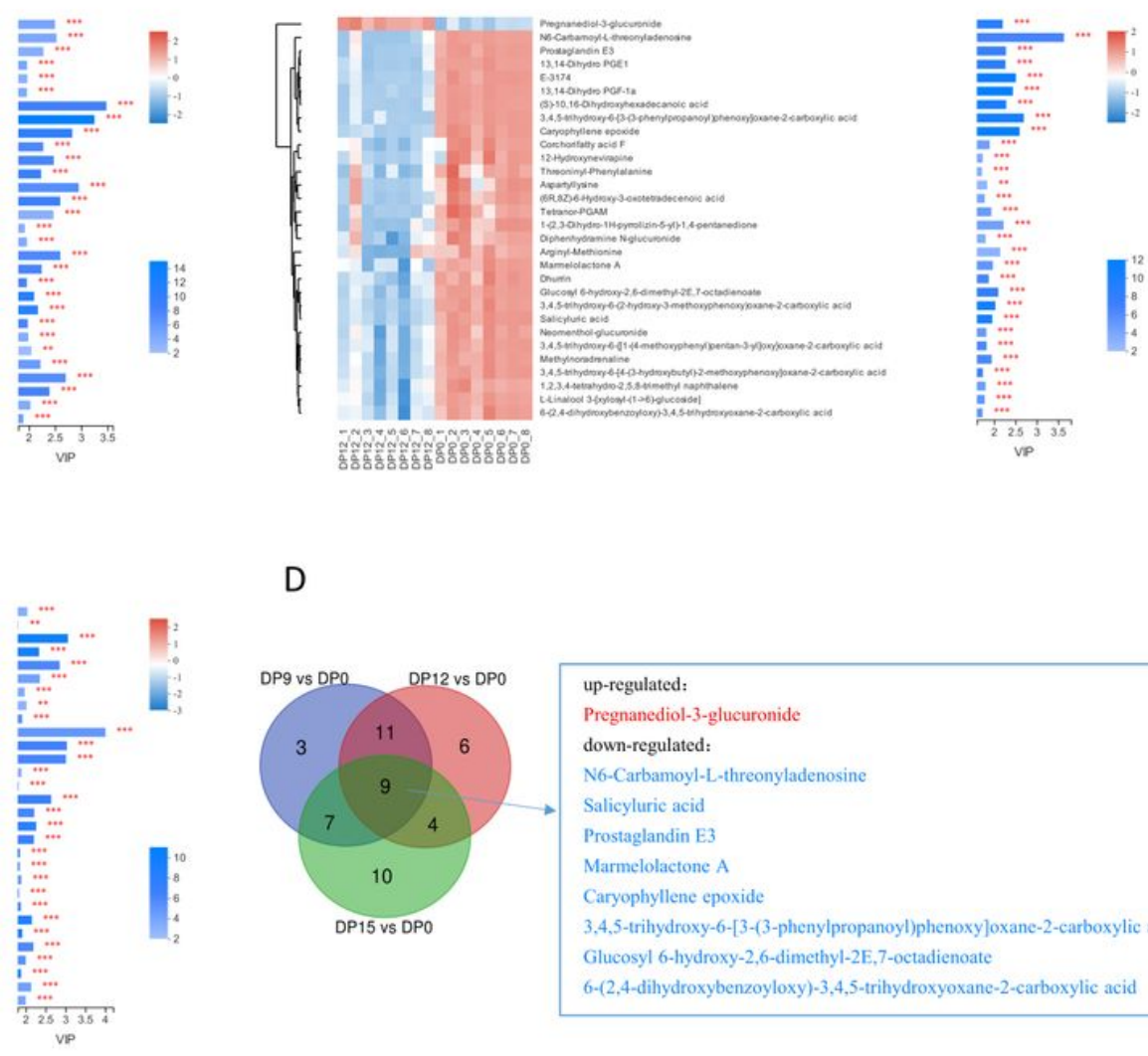

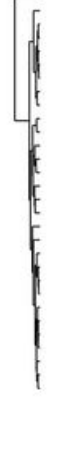

D

Expression Profile \& VIP of Metabolites

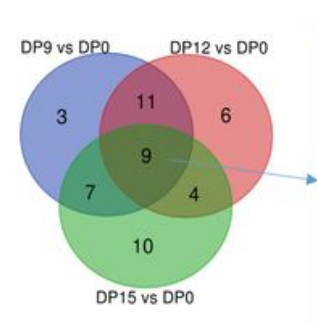

\section{Figure 4}

VIP scores analysis based on weighted coefficients of the OPLS-DA model used to rank the contribution of top 30 metabolites to the discrimination between estrus and early pregnancy groups. (A) Heat map showing VIP of Metabolites between DP9 and DP0, (B) A heat map showing VIP of Metabolites between DP12 and DP0, (C) A heat map showing VIP of Metabolites between DP15 and DP0, (D) The overlap of early pregnancy (DP9, DP12, DP15) and estrus (DP0).
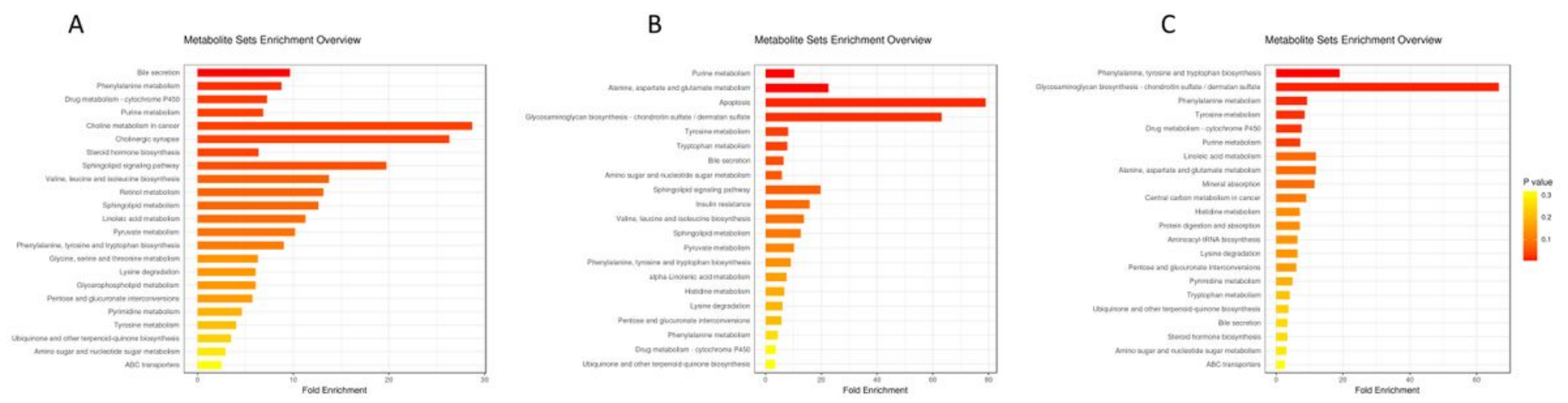

Figure 5 
Histogram showing differential metabolites annotated by comparison to the Kyoto Encyclopedia of Genes and Genomes (KEGG) database. Differential metabolites were classified by KEGG pathway enrichment and significance analysis. Fold enrichment is presented as the ratio of the number of metabolites assigned to the modified pathway by enrichment analysis to the theoretical number of metabolites assigned to the modified pathway by random distribution. The degree of enrichment is indicated by different colors in the histogram according to the p-value. Histogram (A) shows DP9 vs DP0, Histogram (B) shows DP12 vs DP0, whereas histogram (C) shows DP15 vs DP0.

\section{Supplementary Files}

This is a list of supplementary files associated with this preprint. Click to download.

- SupplementaryFigures.pdf

- Tables1.Allthepositiveionfeatures.xls

- TableS2.Allthenegativeionfeatures.xls

- TableS3.Highconfidencemetabolites.xls

- TableS4.MetabsetVipDP9vsDP0.xls

- TableS5.MetabsetVipDP12vsDP0.xls

- TableS6.MetabsetVipDP15vsDP0.xls 